\title{
Mathematical Model and IDPSO Algorithm Research on Dynamic Fleet Planning
}

\author{
Shaojuan $\mathrm{Su}^{1, *}$, Bo $\mathrm{Liu}^{2}$ and Hualin $\mathrm{Wang}^{3}$ \\ ${ }^{I}$ Transportation Equipment and Ocean Engineering College, Dalian Maritime University, Dalian Liaoning, China \\ ${ }^{2}$ Dalian Shipping College, Dalian 116026, China \\ ${ }^{3}$ Dalian Shipbuilding Industry Offshore CO. Ltd, Dalian Liaoning, 116026, China
}

\begin{abstract}
A mathematical model for dynamic fleet planning with multi routes and multi ship, which took the maximum total operation profits as its objective function, was built up according to the characteristics of the shipping company. The model had the following characteristics: Firstly in the model, the long-term fleet planning and short-term scheduling were combined. Secondly, the economic and technology indexes were changed with ship's age, which fully reflect the dynamic fleet planning. At the same time, because of being at a large scale, discrete, multi-dimensional and multi-stage optimization problem has become very difficult to solve. An improved discrete particle swarm optimal (IDPSO) algorithm was used to solute the model. According to the characteristics of the traditional discrete particle swarm optimization algorithm, some methods including coding strategy, iterative formula, initialization, dimension mutation operator to avoid precocious etc. for discrete particle swarm algorithm, were used to improve the method, which can be more suitable for fleet planning's mathematical model and to avoid premature phenomenon. Finally, an example was given to show that the improved method was effective.
\end{abstract}

Keywords: Discrete particle optimal swarm algorithm, dynamic, fleet planning, mathematical model, optimization.

\section{INTRODUCTION}

Over time, inevitably some old ships will gradually be eliminated, scraped, and placed out of service due to the change of technical statuses or operating conditions but new ships will also be added to the fleet in order to maintain the stable development of the transportation fleet in the process of operation and development. This issue must be solved for fleet planning. Fleet planning is in direct relation to the economic benefit and the overall structure of the enterprise. And it also affects the enterprise's competitive, survival and development ability. Large shipping companies have been involved in this study. The current planning has two main disadvantages from the point of view of shipping's practical considerations. First, the current method does not consider the change of ship technical and economic index with age. Secondly the mathematical model cannot solve the problem of long-term planning for a large shipping enterprise. Based on the above, multi-object dynamic fleet planning model is in focus [1].

\footnotetext{
*Address correspondence to this author at the Dalian Linghai Road No. 1, Postcard: 116026, China; Tel: 86-18940815072; E-mail: katie306@163.com
}

\section{MATHEMATICAL MODEL FOR FLEET PLANNING}

In order to make the mathematical model approach to the actual problems, firstly description and hypothesis about ship operating conditions are suggested $[2,3]$.

The number of routes is $\mathrm{G}$. The ships can fully load cargo at the loading ports and unload the full cargo at the unloading port and then return to the loading port. That is to say, the ships are transporting good by a simple mode.

There are several types of ships in the fleet which sail in multi routes;

The planning period for the sail is $\mathrm{N}$ years from 0 year to the end of N-1 years;

The ship's idle capacity range appropriation is selected according to the actual situation of the shipping companies.

By solving the mathematical model, optimal decision for the following variables is needed to be given [4]:

1) Optimal ship scheme for routes each year is needed;

2) If the capacity is lacking, then how many ships need to be increased every year?

3) If the capacity is in excess, which ships will be idle and how many will be idle every year. 


\subsection{Mathematical Model}

Object Function $[5,6]$

$$
\begin{aligned}
& \max Z=\max \left(\sum_{t=0}^{N-1}\left(1+i_{0}\right)^{-(t-1)} Z_{t}\right) \\
& Z_{0}=\left\{\sum_{j=1}^{G} \sum_{i=1}^{K_{0}} A P_{i, j 0} \cdot x_{i, j 0}-\sum_{i=1}^{K} p_{i 0} \cdot F_{i, 0}\right\}+\left\{\sum_{j=1}^{G} \sum_{i=1}^{K} A P_{i j 0} \cdot x_{i j 00}-\sum_{i=1}^{K} p_{i 00} \cdot F_{i 0}\right\} \\
& Z_{1}=\left\{\sum_{j=1}^{G} \sum_{i=1}^{K_{0}} A P_{i, j} \cdot x_{i, j 1}-\sum_{i=1}^{K} p_{i, 1} \cdot F_{i, 1}\right\}+\left\{\sum_{j=1}^{G} \sum_{i=1}^{K} A P_{i j 1} \cdot x_{i j 01}-\sum_{i=1}^{K} p_{i 01} \cdot F_{i 1}\right\} \\
& +\left\{\sum_{j=1}^{G} \sum_{i=1}^{K} A P_{i 0} \cdot x_{i j 10}-\sum_{i=1}^{K} p_{i 10} \cdot F_{i 0}\right\} \\
& \left\{Z_{2}=\left\{\sum_{j=1}^{G} \sum_{i=1}^{K_{0}} A P_{i, j 2} \cdot x_{i, j 2}-\sum_{i=1}^{K} p_{i, 2} \cdot F_{i, 2}\right\}+\left\{\sum_{j=1}^{G} \sum_{i=1}^{K} A P_{i j 2} \cdot x_{i j 02}-\sum_{i=1}^{K} p_{i 02} \cdot F_{i 2}\right\}\right. \\
& +\left\{\sum_{j=1}^{G} \sum_{i=1}^{K} A P_{i j 1} \cdot x_{i j 11}-\sum_{i=1}^{K} p_{i 11} \cdot F_{i i}\right\}+\left\{\sum_{j=1}^{G} \sum_{i=1}^{K} A P_{i j 0} \cdot x_{i j 20}-\sum_{i=1}^{K} p_{i 20} \cdot F_{i 0}\right. \\
& Z_{N-1}=\left\{\sum_{j=1}^{G} \sum_{i=1}^{K_{0}} A P_{i, j(N-1)} \cdot x_{i j(N(N-1)}-\sum_{i=1}^{K} p_{i,(N-1)} \cdot F_{i_{1}(N-1)}\right\}+\left\{\sum_{j=1}^{G} \sum_{i=1}^{K} A P_{i j(N-1)} \cdot x_{i j 0(N-1)}\right. \\
& \left.-\sum_{i=1}^{K} p_{i(0(N-1)} \cdot F_{i(N-1)}\right\}+\cdots+\left\{\sum_{j=1}^{G} \sum_{i=1}^{K} A P_{i j 0} \cdot x_{i(N(N-1))}-\sum_{i=1}^{K} p_{i(N-1))} \cdot F_{i 0}\right\} \\
& i=1,2, \cdots, K ; j=1,2, \cdots G ; t=0,1, \cdots N-1 ; i_{1}=1,2, \cdots K_{0}
\end{aligned}
$$

Constraint conditions $[7,8]$

1) Freight volume in the route cannot be greater than the demand cargo

$$
\left\{\begin{array}{l}
\sum_{i=1}^{K_{0}} Q_{i, j 0} \cdot x_{i, j 0}+\sum_{i=1}^{K} Q_{i j 0} \cdot x_{i j 00} \leq W_{j 0} \\
\sum_{i=1}^{K_{0}} Q_{i, j 1} \cdot x_{i, j 1}+\sum_{i=1}^{K} Q_{i j 1} \cdot x_{i j 01}+\sum_{i=1}^{K} Q_{i j 0} \cdot x_{i j 10} \leq W_{j 1} \\
\sum_{i=1}^{K_{0}} Q_{i, j 2} \cdot x_{i, j 2}+\sum_{i=1}^{K} Q_{i j 2} \cdot x_{i j 02}+\sum_{i=1}^{K} Q_{i j 1} \cdot x_{i j 11}+\sum_{i=1}^{K} Q_{i j 0} \cdot x_{i j 20} \leq W_{j 2} \\
\vdots \\
\vdots \\
\sum_{i=1}^{K_{0}} Q_{i, j(N-1)} \cdot x_{i, j(N-1)}+\sum_{i=1}^{K} Q_{i j(N-1)} \cdot x_{i j 0(N-1)}+\cdots+\sum_{i=1}^{K} Q_{i j 0} \cdot x_{i j(N-1) 0} \leq W_{j(N-1)} \\
i=1,2, \cdots K ; i_{1}=1,2, \cdots K_{0} ; j=1,2, \cdots G ; t=0,1, \cdots N-1
\end{array}\right.
$$

2) The fleet's continuous development constraints

The number of existing ships is $i_{1}$ that are used on every route. The number of idle ship every year is equal to the number of ships $i_{1}$ minus the retired ship that have just entered the study period.

$$
\left\{\begin{array}{l}
\sum_{j=1}^{G} x_{i_{1} j 0}+p_{i_{1} 0}=A_{i_{1}}-W T_{i_{1} 0} \\
\sum_{j=1}^{G} x_{i_{j} j 1}+p_{i_{1} 1}=A_{i_{1}}-W T_{i_{1} 1} \\
\vdots \\
\sum_{j=1}^{G} x_{i_{1} j(N-1)}+p_{i_{1}(N-1)}=A_{i_{1}}-W T_{i_{1}(N-1)}
\end{array}\right.
$$

At the beginning, the buying ships are represented from $i$ and the number is allocated to the route and idle is equal to the number of buying ships.

$$
\left\{\begin{array}{l}
\sum_{j=1}^{G} x_{i j 00}+p_{i 00}=C_{i 0} \\
\sum_{j=1}^{G} x_{i j 01}+p_{i 01}=C_{i 0} \\
\vdots \\
\sum_{j=1}^{G} x_{i j 0(N-1)}+p_{i 0(N-1)}=C_{i 0}
\end{array}\right.
$$

In the first year the number of ships to be bought is $i$. This number is allocated to the route and the idle ship number is equal to the number of the bought ships.

$$
\left\{\begin{array}{l}
\sum_{j=1}^{G} x_{i j 10}+p_{i 10}=C_{i 1} \\
\sum_{j=1}^{G} x_{i j 11}+p_{i 11}=C_{i 1} \\
\vdots \\
\sum_{j=1}^{G} x_{i j 1(N-2)}+p_{i 1(N-2)}=C_{i 1}
\end{array}\right.
$$

In $\mathrm{N}-2$ year the number of ships to be bought is $i$ this number is allocated to the route and number of idle ships is equal to the number of ships to be bought.

$$
\left\{\begin{array}{l}
\sum_{j=1}^{G} x_{i j(N-2) 0}+p_{i(N-2) 0}=C_{i(N-2)} \\
\sum_{j=1}^{G} x_{i j(N-2) 1}+p_{i(N-2) 1}=C_{i(N-2)}
\end{array}\right.
$$

In $\mathrm{N}-1$ year number of ships bought is $i$. This number is allocated in the route and the number of idle ships is equal to the number of ships bought.

$\sum_{j=1}^{G} x_{i j(N-1) 0}+p_{i(N-1) 0}=C_{i(N-1)}$

3) Non-negative integer's variables constraints teger

$x_{i, j t} \geq 0 ; p_{i, t} \geq 0 x_{i j t n} \geq 0 ; p_{i t n} \geq 0 ; C_{i t} \geq 0$ and they are in-

$i=1,2, \cdots K \quad j=1,2, \cdots G ; t=0,1,2, \cdots N-1 ; n=0,1, \cdots N-1 ;$

$i_{1}=1,2, \cdots K_{0}$

In the functions:

$Z-$ Is the objective function

$x_{i_{1}, j}$-is the decision variable, the number of the ships which run on the route $j$ in the $t$ year. At the same time the ship $i_{1}$ refers to the ship before entering the planning period. 
$p_{i_{1} t}$-is the decision variable, the number of idle ships $i_{1}$ in the $t$ year. At the same time the ship $i_{1}$ refers to the ship before entering the planning period.

$x_{i j t n}$-is the decision variable, the number of ships $i$ which run on the route $j$ in the $n$ year. At the same time the ship $i$ refers to the ship buying in the $t$ year.

$p_{i t n}$-is the decision variable, the number of idle ships $i$ in the $n$ year. At the same time the ship $i$ refers to the ship buying in the $t$ year.

$C_{i t}$-is the decision variable, the number of ships $i$ bought in the ${ }^{t}$ year.

$A P_{i, j t}$ - The single ship annual profit for the ship $i_{1}$ running on route $j$ in the $t$ year. At the same time the ship $i_{1}$ refers to the ship before entering the planning period.

$F_{i, t}$-The idle cost for the ship $i_{1}$ which refers to the ship before entering the planning period.

$Q_{i, j t}$ - The single ship annual cargo for the ship $i_{1}$ running on route $j$ in the $t$ year. At the same time the ship $i_{1}$ refers to the ship before entering the planning period.

$A P_{i j t}$ - The single ship annual profit for the ship $i$ running on route $j$ in the $t$ year. At the same time the ship $i$ refers to the ship bought in the $t$ year.

$F_{i t}$-is the idle cost for the ship $i$ which refers to the ship bought in the $t$ year.

$Q_{i j t}$-is the single ship annual cargo for the ship $i$ running on route $j$ in the $t$ year. At the same time the ship $i$ refers to the ship bought in the $t$ year.

$W_{j t}$-is the demand cargo on the route $j$ in the $t$ year.

$A_{i 1}$-is the number of ship $i_{1}$ before entering the planning period.

$W T_{i_{1} t}$-is the number of idle ship $i_{1}$ in the $t$ year.

$i_{0}$ - is the discount rate of considering the fund time value.

$\mathrm{K}$ - is the number of ship pre-added

$\mathrm{G}$ - is the number of routes;
$K_{0}$ - Is the total number of ship types;

\subsection{Model Description}

In this paper we have considered that the economic object changes with time that is more close to the actual operating conditions. Fleet planning model (1) (8) can not only solve the optimal fleet purchase planning over the next several years but also gives ship routing scheme and idle capacity each year in planning. The results are intuitive, concrete, and meet the actual needs of fleet planning and ship operation organization. Fleet planning problem is a complex combinatorial optimization problem. It has large, discrete and integral characteristics. When adopting the dynamic programming method, state variables are growing exponentially and computing speed is decreased with the increase of the stage number and ship type number, so that the "dimension disaster" appears. The application of DPSO to multi object dynamic fleet planning is adopted in this paper. This application aims to explore its feasibility and effectiveness and provides a new idea for a large-scale fleet planning.

\section{DPSO APPLICATION IN THE FLEET PLAN- NING}

\subsection{Introducing the Basic Principle}

PSO algorithm comes from the simulation of the bird prey behavior. A flock of birds search for food in a random order. If there is only a piece of food, the most simple and effective strategy to find food is to search for the area around the birds which is nearest to the food. The PSO algorithm and genetic algorithm are similar and are based on the group and fitness concept. The position of the particle represents the possible solutions of the fitness of the particle to measure the quality of particles. $M$ particles form a community in which the particles are represented as vectors in a D dimension $X_{i}=\left(x_{i 1}, x_{i 2}, \cdots, x_{i D}\right)^{T}$, in a D dimensional search space. The position of the particles $i$ in the $\mathrm{D}$ dimensional search space is $x_{i}$. In other words, the position of each particle is a potential solution. Bring $x_{i}$ into an objective function to calculate the fitness value then according to the fitness value to measure $x_{i}$. The fly speed of the particle $i$ is $V_{i}=\left(v_{i 1}, v_{i 2}, \cdots, v_{i D}\right)$. Record the optimal position of the particles search far from $p_{i}=\left(p_{i 1}, p_{i 2}, \cdots, p_{i D}\right)^{T}$ and the global extreme value is recorded as $g_{g}=\left(p_{g 1}, p_{g 2}, \cdots, p_{g D}\right)^{T}$. Particles renew the position and velocity on their own according to the following two formulas after finding the two extreme values $[9,10]$.

$v_{i d}^{k+1}=\omega v_{i d}^{k}+c_{1} \cdot r_{1} \cdot\left(p_{i d}^{k}-x_{i d}^{k}\right)+c_{2} \cdot r_{2} \cdot\left(g_{g d}^{k}-x_{i d}^{k}\right)$ 
$x_{i d}^{k+1}=x_{i d}^{k}+v_{i d}^{k+1}$

In the formula, $i=1,2, \cdots, m ; d=1,2, \cdots, D$; acceleration constants $c_{1}$ and $c_{2}$ are not less than zero. $r_{1}$ and $r_{2}$ are random numbers between $[0,1] ; ; v_{i d} \in\left[-v_{\max }, v_{\max }\right] v_{\max }$ is a constant and set by the user. $k=1,2, \cdots$ is the iterations number. Formulas (9) and (10) are composed of the initial POS algorithm.

Kennedy and Eberhart [11] put forward Discrete Particle Swarm Optimization (DPSO). In the model $x_{i d}$ was limited to 1 or 0 , while the velocity was not limited but has the probability to change the particle's position

The specific algorithm is as follows:

$$
\begin{aligned}
& S\left(v_{i d}^{k+1}\right)=\frac{1}{1+e^{v_{i d}^{k+1}}} \\
& \text { If } S\left(v_{i d}^{k+1}\right)<\rho \begin{cases}\text { then } & x_{i d}^{k+1}=1 \\
\text { else } & x_{i d}^{k+1}=0\end{cases} \\
& \rho \text { is a random number between }[0,1] .
\end{aligned}
$$

\subsection{The Improved DPSO Method}

Although the DPSO method can effectively solve the discrete problems, yet it is also easy to fall into premature convergence and local minimum. A method to solve this problem is to expand the scale of the particle swarm. This method can only improve the performance to a certain extent, but it cannot fully overcome the premature convergence. At the same time it will also increase the amount of calculation.

The phenomena will be observed in the analysis process of DPSO when it is being used to solve the discrete particle swarm premature convergence problem.

1) When some particles reach the local optimal solution, the rest of the particles quickly close around the position. After which a large number of particles will stay in this position, and after a period of time particle swarm aggregation will occur.

2) Because a large number of particles stay at the local optimal solution position, switching operations don't occur, as a result these particles that stop at the local optimal solution and lose the ability to search. Discrete particle swarm algorithm was improved according to the characteristics of fleet planning problem [12-15].

1) The iterative formula

In the process of implementing the algorithm, the corresponding position of the particle is an integer matrix and speed is a real matrix. The optimization process has both discrete variables and continuous variables. Because of real coding in this paper, the binary particle swarm optimization calculation iterative formula is adopted but is slightly modified in order to calculate the iteration. The specific modification method is the following (particle $i$ as an example):

$$
\left\{\begin{array}{l}
x_{i j}^{(t)}(k+1)-\operatorname{int}\left(x_{i j}^{(t)}(k+1)\right) \geq 0.5 \\
\text { then } x_{i j}^{(t)}(k+1)=\operatorname{int}\left(x_{i j}^{(t)}(k+1)\right)+1 \\
x_{i j}^{(t)}(k+1)-\operatorname{int}\left(x_{i j}^{(t)}(k+1)\right)<0.5 \\
\text { then } x_{i j}^{(t)}(k+1)=\operatorname{int}\left(x_{i j}^{(t)}(k+1)\right)
\end{array}\right.
$$

When $v_{i j}^{(t)}(k+1)$ and $x_{i j}^{(t)}(k+1)$ beyond its range, its value is given according to boundary [14].

2) Initialization

According to the characteristics of fleet planning (which enables the selected solutions to meet the transportation and idle tasks as little as possible), randomly select $N_{0}$ feasible solutions. The fitness is calculated for every feasible solution. Then, $N\left(N<N_{0}\right)$ is selected, as it is the better feasible solution found for the initial population.

3) To avoid premature convergence [15]

Particle swarm algorithm early search speed is very fast. But later on when all the particles tend to move towards historically best position $P_{g}$, the velocity of particles will mainly be decided by the first of types (9). Because $\omega$ is less than 1 , the velocity of particles decreases, and all the particles tend to be of the same diversity. Aiming at the shortcoming of PSO, scholars have put forward the variation theory, which says that the particle swarm optimization results are unchanged after M's iterations. According to the probabilistic $P_{m}$, mutation operation is used to let the new particles, which are randomly generated, replace the original particles. The diversity of the particles can regain in the iteration later on by variation. The variation in the particle is from "death" to the "rebirth" process. It is actually a full dimensional variation, namely old particle information is completely eliminated. The convergence speed in the iteration's late period can be accelerated by full dimensional variation but sometimes will still be trapped in the local optimal. Next position of particles is decided together by the current position and the current speed. The value of the speed decides the flying distance and speed direction decides direction in which to go. The current velocity of particles is determined by 3 factors: the original speed, individual extremum $p_{i d}$ and global extremum $p_{g d}$. If the algorithm has premature convergence, $p_{g d}$ must be a local optimal solution. The particles can go to other areas for research by changing $p_{g d}$. The algorithm is likely to find the global optimal solution. Therefore, when the position of the optimal solution ( $\left.p_{g d}\right)$ doesn't change or change very little, the position $p_{g d}$ is retained. Dimensional variation is implemented on $p_{g d}$ and replaced $p_{g d}$.

The specific steps are as follows:

(1) To determine the dimensions of variation $m(m \prec D)$. 
Table 1. The existing main ship type and number.

\begin{tabular}{|c|c|c|c|}
\hline Ship (t) & Ship's Age & Ship Price (Million Yuan) & Number \\
\hline \hline 6000 & 16 & 2196 & 7 \\
\hline 9500 & 0 & 4500 & 2 \\
\hline 16000 & 9 & 40633 & 2 \\
\hline 37000 & 20 & 4750 & 2 \\
\hline 43000 & 18 & 17000 & 1 \\
\hline 54000 & 20 & 4419 & \\
\hline
\end{tabular}

(2) After the end of the iteration, randomly generate different integration $u_{1}, u_{2}, \cdots, u_{m}$ in $[0, D]$.

(3) Mutate $p_{g d}$ in the position of $u_{1}, u_{2}, \cdots, u_{m}$ to get a new vector Mbest.

(4) Calculate the fitness $f_{1}$ and $f_{2}$ of Mbest and $p_{g d}$.

(5) If $f_{1} \prec f_{2}$ Then, repeat steps (1) to (4) until reach to the iteration number. If $f_{1}$ is still less than $f_{2}$ then $p_{g d}$ is the optimal solution otherwise Mbest $=p_{g d}$.

\subsection{Calculation Process}

(1) Initialize $N$ feasible solutions to form the initial population by using this method and initialize the particle velocity. The position and velocity of the particle can be represented by the following matrix:

$X_{i}=\left[\begin{array}{lcccc}x_{i 1}^{(0)} & x_{i 2}^{(0)} & x_{i 3}^{(0)} & \cdots & x_{i m}^{(0)} \\ x_{i 1}^{(1)} & x_{i 2}^{(1)} & x_{i 3}^{(1)} & \cdots & x_{i m}^{(1)} \\ \vdots & \vdots & \vdots & \cdots & \vdots \\ x_{i 1}^{(t)} & x_{i 2}^{(t)} & x_{i 3}^{(t)} & \cdots & x_{i m}^{(t)} \\ \vdots & \vdots & \vdots & \cdots & \vdots \\ x_{i 1}^{(N-1)} & x_{i 2}^{(N-1)} & x_{i 3}^{(N-1)} & \cdots & x_{i m}^{(N-1)}\end{array}\right]$

$V_{i}=\left[\begin{array}{lcccc}v_{i 1}^{(0)} & v_{i 2}^{(0)} & v_{i 3}^{(0)} & \cdots & v_{i m}^{(0)} \\ v_{i 1}^{(1)} & v_{i 2}^{(1)} & v_{i 3}^{(1)} & \cdots & v_{i m}^{(1)} \\ \vdots & \vdots & \vdots & \cdots & \vdots \\ v_{i 1}^{(t)} & v_{i 2}^{(t)} & v_{i 3}^{(t)} & \cdots & v_{i m}^{(t)} \\ \vdots & \vdots & \vdots & \cdots & \vdots \\ v_{i 1}^{(N-1)} & v_{i 2}^{(N-1)} & v_{i 3}^{(N-1)} & \cdots & v_{i m}^{(N-1)}\end{array}\right]$

$X_{i}$--- The particle $i ; x_{i j}^{t}$ - the number of the ship $j$ which is bought at the planning stage $t$; $v_{i j}^{t}$ is randomly are generated in the interval $\left[v_{j \max d}^{t}, v_{j \min d}^{t}\right]$.
(2) The optimal position of each particle $p_{i}=\left(p_{i 1}, p_{i 2}, \cdots p_{i n}\right)$ is the particle location now. The corresponding fitness $p$ Best is calculated. The global optimum location $p_{g}=\left(p_{g 1}, p_{g 2}, \cdots p_{g n}\right)$ is the position of the particle with the best fitness and gbest is the fitness.

(3) $\mathrm{k}=\mathrm{k}+1$.

(4) For every particle in the swarm, preform the following steps: (1) according to equations (9), (10) and (11) update the position and velocity of particles and calculate the fitness; (2) if the fitness of $i$ is better than its corresponding pBest, then $p_{i}=\left(p_{i 1}, p_{i 2}, \cdots p_{i n}\right)$ is set as the particle's new position and update pBest . (3) if there is a fitness of a particle, which is better than the optimal adaptive fitness of particles then set the position of the particle to $p_{g}=\left(p_{g 1}, p_{g 2}, \cdots p_{g n}\right)$ and update gbest and return to (3).

(5) If after M time's successive iterations, particle swarm optimization result doesn't remain unchanged, select the particle which is not the optimal particle to mutate according to probabilistic $P_{m}$. Let the new particles, which have been randomly generated, to replace the original particles, and then return to (3).

(6) The maximum number of iterations is reached and part dimensional variability.

(7) Output results

The calculation process is as follows in Fig. (1).

\section{EXAMPLE}

An ore transport fleet planning problem in the next five years is researched in the example. The fleet has six types of ships as shown in Table 1. In addition there are seven new types of ships which are to be made available for future as shown in Table 2. The freight volume of the route is shown in Table 3.

The discrete particle swarm optimization algorithm is used to calculate the fleet planning problem. This process makes the swarm size up to 30 , and the particle mutation 


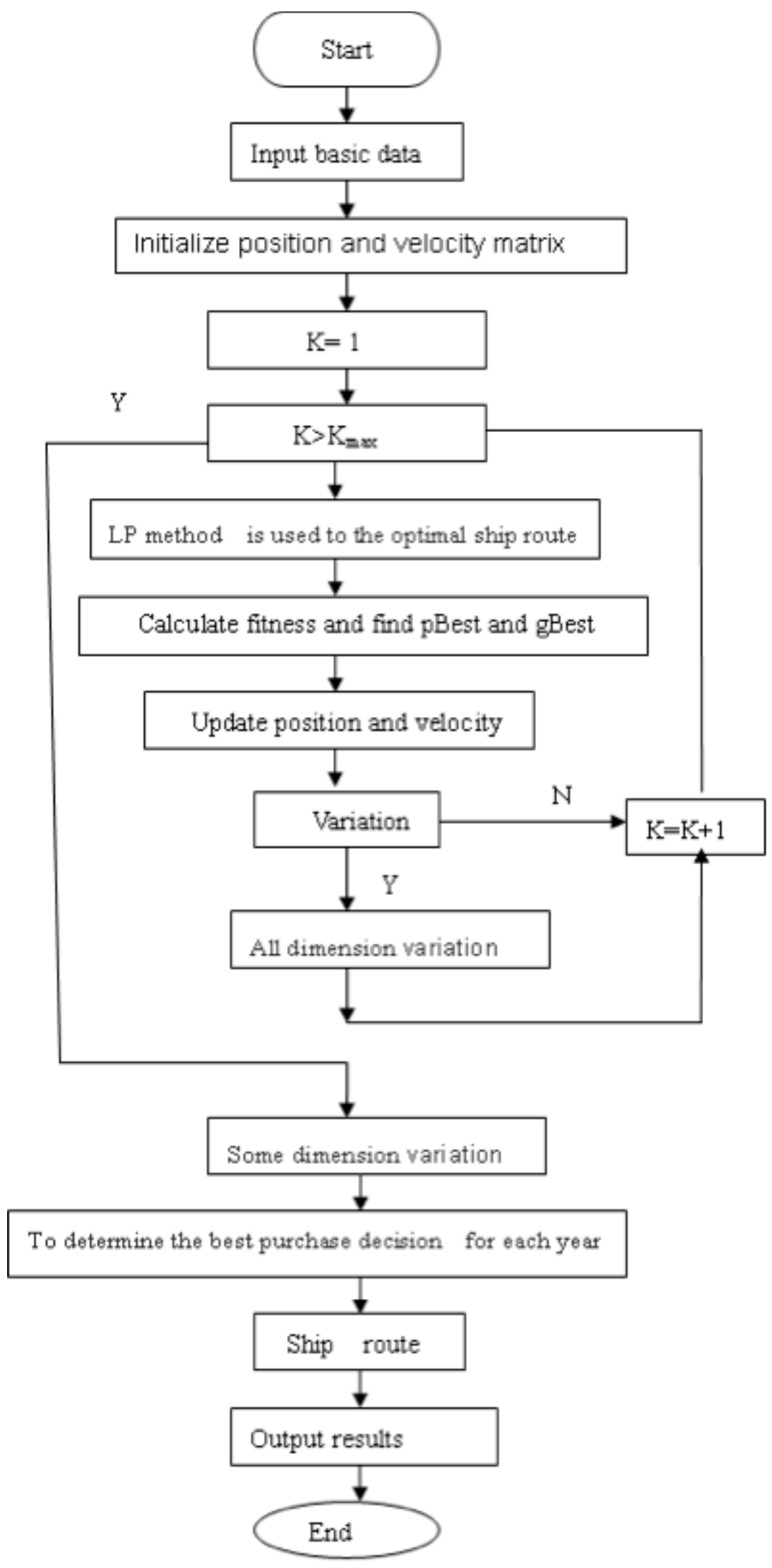

Fig. (1). Calculation process. 
Table 2. Main parameters of typical ships.

\begin{tabular}{|c|c|c|c|c|c|c|c|c|}
\hline Ship & $\begin{array}{l}\mathbf{L}_{\mathrm{OA}} \\
\text { (m) }\end{array}$ & $\begin{array}{l}\mathbf{L}_{\mathrm{PP}} \\
\text { (m) }\end{array}$ & $\begin{array}{c}\text { B } \\
\text { (m) }\end{array}$ & $\begin{array}{c}\text { D } \\
\text { (m) }\end{array}$ & $\begin{array}{c}T \\
\text { (m) }\end{array}$ & $\begin{array}{c}\text { DW } \\
\text { (t) }\end{array}$ & $\begin{array}{c}\mathrm{v} \\
\text { (kn) }\end{array}$ & $\begin{array}{c}\text { HP } \\
(\mathrm{kW})\end{array}$ \\
\hline 2. $0 \mathrm{WT}$ & 164 & 154 & 22 & 13.4 & 9.5 & 19509 & 14 & 5647 \\
\hline 2. $5 \mathrm{WT}$ & 164 & 154 & 24.2 & 14.6 & 9.7 & 23000 & 15 & 5736 \\
\hline 3. $0 \mathrm{WT}$ & 198 & 186 & 24.5 & 14.6 & 10.2 & 30000 & 14 & 6534 \\
\hline $3.5 \mathrm{WT}$ & 186 & 178 & 28.4 & 15.6 & 11.25 & 36000 & 15. & 7942 \\
\hline 4. $0 \mathrm{WT}$ & 195 & 185 & 32 & 15.2 & 10 & 39800 & 14. & 7720 \\
\hline 4. $5 \mathrm{WT}$ & 196 & 188.4 & 32.4 & 16.6 & 11.7 & 49600 & 14 & 6399 \\
\hline 5. $0 \mathrm{WT}$ & 190 & 180 & 32.2 & 16.8 & 11.1 & 50000 & 15 & 6177 \\
\hline
\end{tabular}

Table 3. The volume requirements Unit: million ton/year.

\begin{tabular}{|c|c|c|c|c|c|c|}
\hline Route/Volume & 0 Year Start & 1 Year Start & 2 Year Start & 3 Year Start & 4 Year Start & 5 Year Start \\
\hline 1 & 480 & 544 & 608 & 671 & 736 & 800 \\
\hline 2 & 420 & 476 & 532 & 587 & 644 & 700 \\
\hline 3 & 525 & 595 & 665 & 735 & 805 & 875 \\
\hline 4 & 450 & 510 & 570 & 630 & 690 & 750 \\
\hline 5 & 375 & 425 & 475 & 525 & 575 & 625 \\
\hline 6 & 420 & 476 & 532 & 587 & 644 & 700 \\
\hline 7 & 390 & 442 & 494 & 546 & 598 & 650 \\
\hline
\end{tabular}

Table 4. The best boat purchase plan.

\begin{tabular}{|c|c|c|c|c|c|c|c|}
\hline \multirow{2}{*}{ Year } & \multicolumn{7}{|c|}{ Boat } \\
\hline & 2. $0 \mathrm{wt}$ & $2.5 \mathrm{wt}$ & 3. $0 \mathrm{wt}$ & $3.5 \mathrm{wt}$ & 4. $0 \mathrm{wt}$ & 4. $5 \mathrm{wt}$ & $5.0 \mathrm{wt}$ \\
\hline 0 & 1 & 0 & 0 & 0 & 1 & 0 & 4 \\
\hline 1 & 1 & 0 & 0 & 0 & 0 & 1 & 0 \\
\hline 2 & 0 & 0 & 1 & 0 & 0 & 1 & 0 \\
\hline 3 & 0 & 1 & 0 & 0 & 0 & 0 & 1 \\
\hline 4 & 0 & 0 & 0 & 0 & 2 & 0 & 0 \\
\hline 5 & 0 & 0 & 0 & 0 & 1 & 1 & 0 \\
\hline
\end{tabular}

probability can be denoted by $p_{m}=0.1$, the dimension mutation probability $p_{v}=0.4$, the maximum number of iterations for the $500^{\text {th }}$ time. After 127 iterations, the optimal value can be achieved. Table 4 shows the best boat purchase plan in the next five years for the fleet.
Analysis of the Results:

After the above fleet planning calculation we can find that the tonnage is bigger and the unit transportation cost is lower and profit is higher when the distance and freight volume reached a certain value. It is preferable to buy the ship with a large tonnage. It fully embodies the "long line big 
Table 5. The optimal ship route for the fifth year.

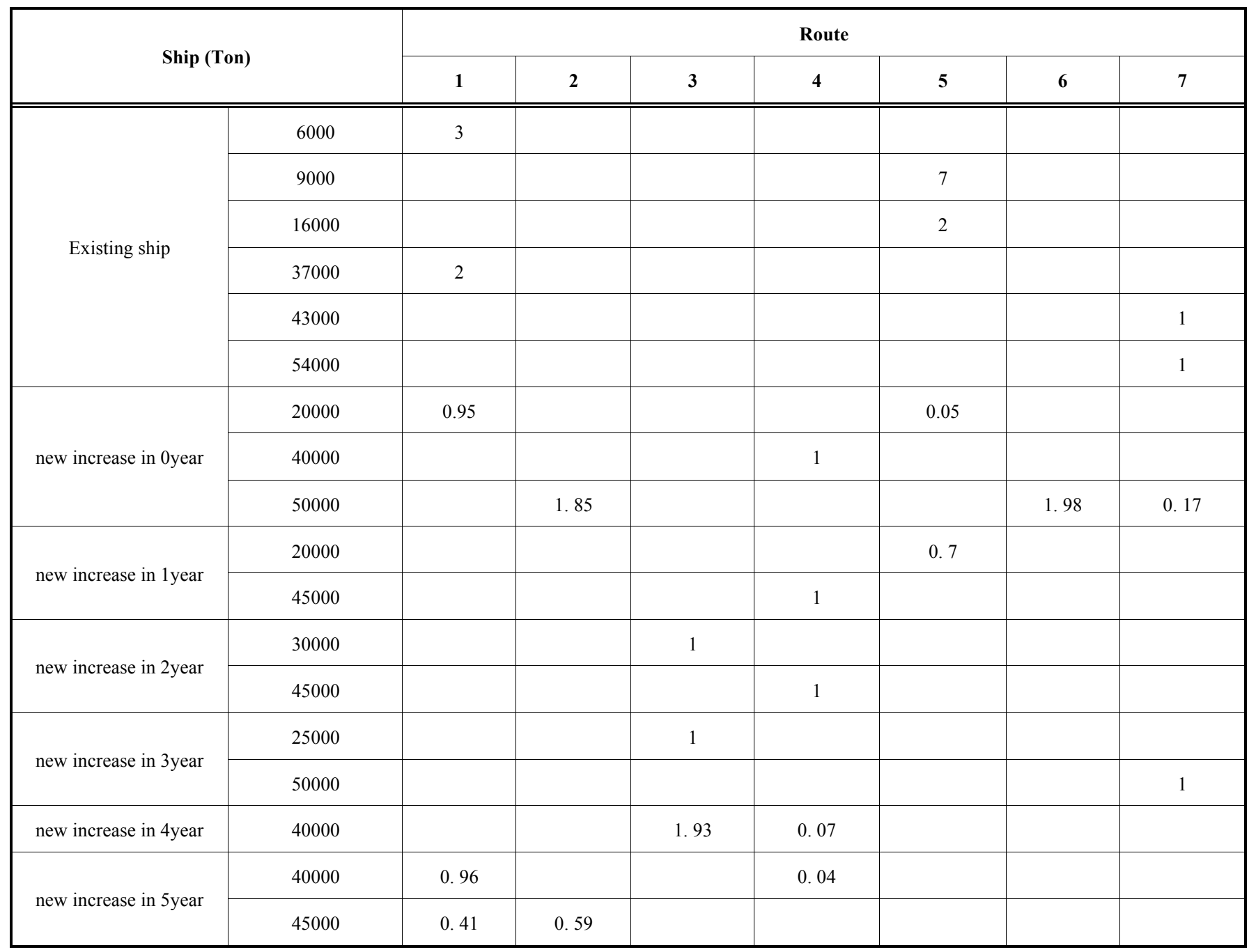

ship" advantage. The purchase plan for the shipping company is to buy, mainly, 40000 tons to 50000 tons in order to increase the capacity under meeting the port and waterway conditions and $20000 \sim 30000$ tons ship as a supplement. Linear programming is used to design the ship route for every year after gaining the purchase scheme. But due to the shortage of space, only fifth year ships routing scheme is mentioned in the paper as shown in Table $\mathbf{5}$.

\section{CONCLUSION}

In this paper, the discrete particle swarm algorithm was carried out to solve the dynamic fleet planning problem. The optimal solution can be quickly given by this method from the actual situation of shipping. This method shows that the proposed algorithm is a feasible method for large-scale, multi-dimensional, multi stage optimization problem. Due to the complexity of the problem itself and computational scale and other reasons, it only takes the profit as the objective function without considering the various elements such as: the technical aspect, timeliness, competitiveness and risk etc.
In future, the calculation speed will be strived to be improved and more objects will be considered into the mathematical model, so that there is more practical value for the shipping enterprises.

In addition, the fleet development planning should be constantly improved with the continuous development of the external environment and enterprise itself to adapt to the outside requirements. Thus, the fleet planning should be recalculated each year or every other year according to the real situation of the transportation market, so that the original planning should be timely modified which is very necessary.

\section{CONFLICT OF INTEREST}

The authors confirm that this article content has no conflict of interest.

\section{ACKNOWLEDGEMENTS}

This work is funded by the Fundamental Research Funds for the Central Universities (3132014068 and 3132014318). 


\section{REFERENCES}

[1] X. Xie, The Ship Scheduling and Fleet Planning Method, Beijing: China Communications Press, 1997.

[2] J. L. Everett, A. C. Hax, V. A. Lewinson, and D. Nudds, "Optimization of a fleet of large tankers and bulkers: a linear programming approach", Marine Tech., pp. 430-438, 1972.

[3] T. A. J. Nicholson, and R. D. Pullen, "Dynamic programming applied to ship fleet management", Oper. Res. Quart., vol. 22 no. 3 pp. 211-220, 1971.

[4] A. N. Perakis, and D. I. Jaramillo, "Fleet deployment optimization for liner shipping, part 1. background, problem formulation and solution approaches", Maritime Policy Manag., vol. 18, no. 3, pp. 183-200, 1991.

[5] K. Rana, and R. G. Vickson, "Routing container ships using lagrangean relaxation and decomposition", Transport. Sci., vol. 25, no. 3, pp. 201-214, 1999.

[6] B. J. Powell, and A. N. Perakis. "Fleet deployment optimization for liner shipping: an integer programming model," Maritime Pol. Manage., vol. 19, no. 3, pp. 33-36, 1997.

[7] X. Xie, H. Sang, and Q. Yang "Case study on fleet planning for carriers of china importing crude oil", Syst. Eng. Theory Pract., vol. 33 , no. 6 , pp. 1544-1549, 2013
[8] S. Su, "Uncertain analytical method research and application on coast dry bulk cargo ships transportation", Wuhan Univ. Technol., pp. 68-99, 2007.

[9] Y. Shi, and R. C. Eerhart, "Particle swarm optimization development applications and resources", In: Proc. Cong. Evol. Comput. NJ: Piscataway, IEEE, pp. 81-86, 2001.

[10] Y. Shi, and R. C. Eerhart, "A modified particle swarm optimizer", In: IEEE World Cong. Comput. Intell., pp. 69-73, 1998.

[11] J. Kennedy, and R. C. Eberhart, "Particle swarm optimization", In: Proceedings IEEE International Conference on Neural Networks, IV Piscataways, NJ Service Center, pp. 1942-1948, 1995.

[12] K. E. Parsopulos, and M. N. Vrahatis, "Recent approaches to global optimization problems through particle swarm optimization", Nat Comput., vol. 1, no. 2-3, pp. 235-306, 2002.

[13] J. Chen, Z. Qin, Y. Liu, and J. Lu, "Particle swarm optimization with local search", Proc. 2005 Int. Conf. Neural Networks and Brain Proc., vol. 1, pp. 481-484, 2005.

[14] Y. Liu, Z. Qin, Z. Xu, and X. He, "Using relaxation velocity update strategy to improve particle swarm optimization", In: Proceedings $3^{\text {rd }}$ International Conference Machine Learning for Cyber, pp. 2469-2472, 2004.

[15] Ning Li, Y. Qin, D. Sun, and T. Zou, "Particle Swarm Optimization with Mutation Operator", In: Proceedings $3^{\text {rd }}$ International Conference Machine Learning for Cyber, 2004, pp. 2251-2256, 2004.

(c) Su et al.; Licensee Bentham Open.

This is an open access article licensed under the terms of the Creative Commons Attribution Non-Commercial License (http://creativecommons.org/licenses/by-nc/4.0/) which permits unrestricted, non-commercial use, distribution and reproduction in any medium, provided the work is properly cited. 